\title{
The yield of lung ultrasound in neonates with respiratory distress
}

\author{
AlZharaa Elsayed Ahmed, Mostafa Ashry Mohammed,
} Mohamad Hasan Alam- Aldeen, Amira Mohammed. Melet. Pediatric Department, Faculty of Medicine, Sohag University, Sohag Egypt.

\begin{abstract}
Background: A systematic ultrasound has been used across a wide span of ages depending on both chest anatomic structures (ie, the ribs or the pleural line)and artifact images, such as the B-lines. The presence of multiple bilateral B-lines is suggestive of an interstitial syndrome(eg, pulmonary edema, interstitial pneumonia, diffuse parenchymal lung disease). In the neonate, the aerated dry lung, which appears as a uniform hypoechogenic pattern, sliding with respiration and showing horizontal reverberations of the pleural image (the A-lines), can be easily differentiated from the hyperechogenic "white" pattern of the "wet" or inflamed organ.

Objective To study the value of chest ultrasound in neonates with respiratory distress and to study the value of chest ultrasonography in comparison with chest $\mathrm{x}$-rays.

Methods: This prospective hospital-based study was conducted on 74 neonates (October 2017to September 2018 in the Neonatal intensive care unit at Sohag university hospital with any degree of respiratory distress. All cases were involved with the physical examination, chest x-ray, and chest ultrasound. Finally, data were analyzed by SPSS software.

Results: In all the 74 cases chest ultrasound was applied and the disease was further categorized into respiratory distress syndrome in 25 cases (33.78\%).Pneumonia in 13 cases $(17.56 \%)$, Transient tachypnea of newborn (TTN) in 25 cases $(33.7 \%)$, Meconium aspiration (MAS), in 9cases(12.16\%).Pleural effusion in 4 cases (5.40\%). We found that there no difference between the diagnosis of the cases by clinical presentation versus chest sonar diagnosis $(p$ value $=0.157$ ) which was statistically insignificant.

Conclusion: no difference between the diagnosis of the cases by (clinically, history, and chest $\mathrm{X}$-rays ) versus chest sonar diagnosis.

Study Limitation: Age group: we include only the neonatal group suffered from the limited disease as RDS, TTN, MAS, Pneumonia, excluding cases with, BBD or those with congenital malformation. The number of a patient: we included only74 patient in our study.Lack of material potential as availability of other facilities like CT scan and lack of experience.
\end{abstract}

Keywords:chest ultrasound in neonates, neonatal respiratory distress, chest ultrasound versus chest $\mathrm{x}$ rays.

\section{Introduction:}

Chest ultrasound has recently been applied with success in different settings of adult medicine, ranging from cardiopulmonary emergencies and trauma surgery(2). A systematic ultrasound has been validated across a wide span of ages using both chest anatomic structures (ie, the ribs or the pleural line)and artifact images, such as the B-lines, that are discrete, laserlike vertical hyperechoic reverberations arising from the pleural line. Multiple diffuse bilateral B-lines are suggestive of an interstitial syndrome(eg, pulmonary edema, interstitial pneumonia, diffuse 
parenchymal lung disease) $(3,4)$. In neonates, the aerated dry lung, appears as a uniform hypoechogenic pattern, sliding with respiration and often showing horizontal reverberations of the pleural image (the A-lines), can be easily differentiated from the hyperechogenic "white" pattern of "wet" or inflamed organ. Noninvasive ventilation (NIV) such as CPAP is used for the treatment of moderate respiratory distress of the newborn to stabilize breathing while avoiding the complications of invasive ventilation. (5). NIV-assisted infants are more easily accessed by parents and health caregivers, allowing an optimization also of note ventilatory strategies. These noninvasive ventilated neonates need regular clinical monitoring for fear of increasing work of respiration or deterioration of their blood gas exchange that requires more invasive endotracheal intubation, surfactant administration, and mechanical ventilation(5)..the conventional chest $\mathrm{x}$ rays were found to be less helpful in grading the severity of neonatal respiratory distress syndrome. On the other hand, the persistence of a "whitelung" ultrasound image has been shown to correlate with clinical respiratory distress (RD) in preterm infants (6).

\section{Material and methods:}

This was a prospective observational study on patients had manifestation suggesting acute respiratory distress admitted in one year at NICU,sohag university hospital, all patients in this study were subjected to the following Clinical histories focusing on.gestional age, antenatal history, sociodemographic factors. Complete clinical examinations focusing on a general look, chest examination, cardiac examination, and radiological chest $\mathrm{x}$ rays \&lung ultrasound. An oral and written consent was taken from parents of patients included in the study and was approved from ethics scientific committee at Sohag University hospital.

\section{Statistical analysis:}

Data were analyzed using SPSS version 17.0. Qualitative data were expressed as number and percentage. McNemar Test and Mc Nemar-Bowker test were used for comparison between different diagnostic methods and findings. Sensitivity, specificity, positive predictive value, negative predictive value and accuracy of the tested findings were calculated. A 5\% level was chosen as a level of significance in all statistical tests used in the study.

\section{Results:}

In our study, the diagnosis of pulmonary disease was based on medical history, clinical manifestations, clinical examination, the cases were categorized as respiratory distress syndrome ( RDS ) and there number s were27 $(36.48 \%)$. Pneumonia in 13cases (17.5\%), transient tachypnea of neonates in 23 cases $(31.08 \%)$,Meconium aspiration syndrome in 9 cases $(12.16 \%)$,pleural effusion in 2 cases $(2.7 \%)$ table (1).

\begin{tabular}{|l|l|}
\hline DISEASE & NUMBER \\
\hline RDS & $27(36.48 \%)$ \\
\hline Pneumonia & $13(17.5 \%)$ \\
\hline TTN & $23(31.08 \%)$ \\
\hline MAS & $\mathbf{9 ( 1 2 . 1 6 \% )}$ \\
\hline Pleural effusion & $2(2.7 \%)$ \\
\hline
\end{tabular}

Table (1): Disease classification according to clinical examination, $x$ rays, and history.

In the all 74 cases enrolled in our study chest ultrasound was applied and the disease was further categorized according to the chest ultrasound finding into respiratory distress syndrome in 25 cases $(33.78 \%)$ which was further subdivided into type I(white lung) in 20 case(80\%), Type II 
(predominance of $\mathrm{b}$ line ) in 5 cases (20\%) .Pneumonia in13 cases $(17.56 \%)$, Transient tachypnea of newborn (TTN) in 25 cases $(33.7 \%)$, Meconium aspiration (MAS) in 9 cases $(12.16 \%)$.Pleural effusion in 4 cases $(5.40 \%)$. The different ultrasound finding in each disease will be discussed in details in the following pages. We found that there no difference between the diagnosis of the cases by (clinically, history, and chest $\mathrm{x}$-rays ) versus chest sonar diagnosis ( $\mathrm{p}$ value $=0.157$ ) which was statistically insignificant . as will be shown in table (2).

\begin{tabular}{||l|l||}
\hline \hline VARIABILITY & NUMBER (\%) \\
\hline $\begin{array}{l}\text { RDS } \\
\text { Type I(white lung) } \\
\text { Type II (predominance } \\
\text { of b line ) }\end{array}$ & $\left.\begin{array}{l}\mathbf{2 5}(\mathbf{3 3 . 7 8 \%}) \\
\mathbf{5}(\mathbf{8 0 \%})\end{array}\right)$ \\
\hline Pneumonia & $\mathbf{1 3}(\mathbf{1 7 . 5 6 \% )})$ \\
\hline TTN & $\mathbf{2 5}(\mathbf{3 3 . 7 \%})$ \\
\hline $\begin{array}{l}\text { Meconium aspiration } \\
\text { (MAS) }\end{array}$ & $\mathbf{9 ( 1 2 . 1 6 \% )}$ \\
\hline Pleural effusion & $\mathbf{4 ( 5 . 4 0 \% )}$ \\
\hline
\end{tabular}

Table (2): Disease variability by chest ultrasound

\begin{tabular}{|l|l|l|l|}
\hline & $\begin{array}{l}\text { RDS } \\
\mathrm{n}=25\end{array}$ & $\begin{array}{l}\text { Transient } \\
\text { tachypnea } \\
\text { of } \\
\text { new- born } \\
\text { (TTN) } \\
\mathrm{n}=25\end{array}$ & $\begin{array}{l}\text { Pneumonia } \\
\mathrm{N}=13\end{array}$ \\
\hline $\begin{array}{l}\text { Pleural-line } \\
\text { Abnormalities }\end{array}$ & $\mathbf{2 5 ( 1 0 0 \% )}$ & $\mathbf{2 5 ( 1 0 0 \% )}$ & $\mathbf{1 1 ( 8 4 . 6 \% )}$ \\
\hline $\begin{array}{l}\text { Absence of A- } \\
\text { line }\end{array}$ & $\mathbf{2 5 ( 1 0 0 \% )}$ & $\mathbf{2 5 ( 1 0 0 \% )}$ & $\mathbf{1 3 ( 1 0 0 \% )}$ \\
\hline $\begin{array}{l}\text { Pulmonary } \\
\text { Edema or } \\
\text { White Lung } \\
\text { (\%) }\end{array}$ & $\mathbf{2 0 ( 8 0 \% )}$ & $\mathbf{7 ( 2 8 \% )}$ & - \\
\hline $\begin{array}{l}\text { Insterstitial } \\
\text { Syndrome }\end{array}$ & $\mathbf{5 ( 2 0 \% )}$ & $\mathbf{1 8 ( 7 2 \% )}$ & $\mathbf{1 3 ( 1 0 0 \% )}$ \\
\hline $\begin{array}{l}\text { Double Lung } \\
\text { Point (\%) }\end{array}$ & - & $\mathbf{1 7}(\mathbf{6 8 \% )}$ & - \\
\hline $\begin{array}{l}\text { Lung } \\
\text { Consolidation } \\
\text { and Air } \\
\text { Bronchograms } \\
\text { (\%) }\end{array}$ & $\mathbf{2 4 ( 9 6 \% )}$ & - & $\mathbf{1 3 ( 1 0 0 \% )}$ \\
\hline
\end{tabular}

Table (3) Lung ultrasonic manifestations in lung diseases

Where Type I (reveal the presence of white lung). Type II (predominance of b line).
Amira Mohammed. Melet

In our study we used chest the US to find the most specific findings in each disease and we found that the most specific finding in RDS and TTN was Pleural-line Abnormalities that was found in 25 cases (100), Absence of Aline in 25 cases $100 \%$ while the most specific finding in pneumonia was lung Consolidation and air bronchograms that was 13 cases $100 \%$ ) as shown in table (3).

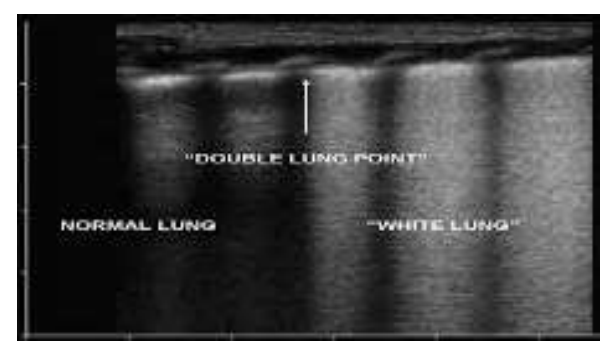

Figure (1)lung consolidation in lung ultrasound

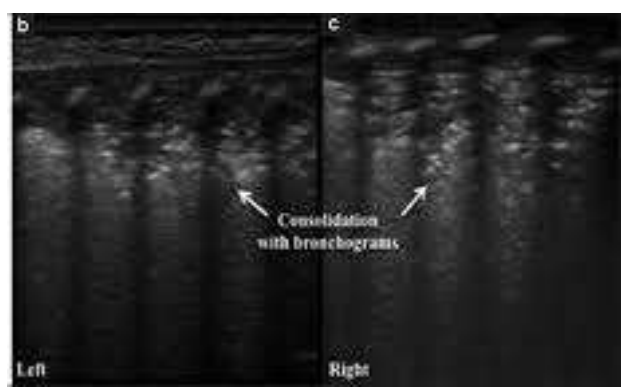

Figure (2) double lung point

\section{Discussion:}

In our study, the cases were categorized as respiratory distress syndrome ( RDS) and their percentage $(36.48 \%)$ transient tachypnea of neonates cases $(31.08 \%)$, Meconium aspiration syndrome $(12.16 \% \mathrm{~s})$, which was higher than those found in. (Shui-Wen Chen. et al.,2017) study which was (19.3), (11.4) and (6.7\%) respectively. Pneumonia (17.5\%) which was less than those found in Shui-Wen Chen. et al.,2017) study which was (29.8\%).

As regard ultrasound results in RDS patients. In our study in case of the total number of RDS diagnosed by chest, the US was $25(83.7 \%)$. The 
abnormalities of the pleural line and Aline disappearance are found in 25 cases (100\%) which matches with. (Shui-Wen et al., 2017) and (Jing et al., 2016) (7,8) while, interstitial syndrome, was present in 0 $\operatorname{cases}(r \cdot \%)$ which was higher than(Shui-Wen et al., 2017) that was $14.8 \%$ and lung consolidation could be found on LUS 24 cases $(96 \%)$ which was less than(Shui-Wen et al., 2017) study that was $100 \%$. pulmonary edema or white lung could be found on LUS $r$. case $(\wedge . \%)$ which was less than(Shui-Wen et al., 2017)). that was $85.2 \%$. This may depend on the clinical severity of the cases. As regards double lung point was zero in our study and this matches with (Shui-Wen et al., 2017).

pleural line abnormality and absence A line was present in (100\%) which matches with (Shui-Wen et al., 2017). While interstitial lung was present in $18(72 \%)$ which was more than (ShuiWen et al., 2017) that was $(70.3 \%)$ as regard pulmonary edema in TTN the total $7(28 \%)$ which was less than (Shui-Wen et al., 2017) which was ( \% ५ . $\vee$ ). This disagreement may be due to the severity of the disease as pulmonary edema observed much in severe cases double lung point was present in 17(68\%) which was more than those found in ( Shui-Wen et al., 2017) and their percent was. (34.1\%).

The total number of pneumonia of diagnosed by chest US was $13(17.56 \%)$ and this was matched with the initial diagnosis made clinically. In our study the abnormalities of the pleural line are found in 11 case $(84.6 \%)$ which was less than ( ShuiWen et al., 2017) that was(90.9\%) . while A-line disappearance and lung consolidation could be found on LUS in all cases of neonatal pneumonia 13 cases $(100 \%)$. Which matches with( Shui-Wen et al., 2017). Double lung point couldn't be seen in pneumonia and this matches with( Shui-Wen et al., 2017) study.

\section{Conclusions:}

no difference between a diagnosis of the cases by (clinically, history, and chest $\mathrm{X}$-rays ) versus chest sonar diagnosis. the most specific finding in RDS and TTN was Pleural-line Abnormalities that was 25 cases (100), Absence of A-line 25 cases $100 \%$ while the most specific finding in pneumonia was Lung Consolidation and Air Bronchograms that was 13 cases $100 \%$ ).

Acknowledgment:

Authors thank colleagues in the Pediatrics Department, Faculty of Medicine, Sohag University (Egypt) for their assistance, and the parents and children who participated in this study. Conflict of Interest:

There is no conflict of interest to be declared.

Authors contributions:

All authors contributed to this project and article equally. All authors read and approved the final manuscript.

\section{References:}

1- Volpicelli G, Caramello V, Cardinale L, Mussa A, Bar F, Francisco MF. Detection of sonographic B-lines in patients with normal lung or radiographic alveolar consolidation. Med Sci Monit 2008; 143: Cr122Cr128.

2- K. Alrajhi, M. Y. Woo, And C. Vaillancourt, "Test characteristics of ultrasonography for the detection of pneumothorax: a systematic review and meta-analysis," Chest, 2012; vol. 141, no . 3, pp. $703-708$,

3- Volpicelli G, Silva F, Radios M. Realtime lung ultrasound for the diagnosis of alveolar consolidation and interstitial syndrome in the emergency department. Eur J Emerg Med. 2010;17(2):63-72

4-Volpicelli G, Elbarbary M, Blaivas M, et al; International Liaison Committee on 
Lung Ultrasound (ILC-LUS) for International Consensus Conference on Lung Ultrasound (ICC-LUS). International evidence-based recommendations for point-of-care lung ultrasound. Intensive Care Med. 2012; 38(4):577-591,.

5- Lista G, Castoldi F, Fontana P, et al. Nasal continuous positive airway pressure (CPAP) versus bi-level nasal CPAP in preterm babies with respiratory distress syndrome: a randomized control trial. Arch Dis Child Fetal Neonatal Ed. 2010;95(2): F85-F89.
6- Copetti R, Cattarossi L, Macagno F, Violino M, Furlan R. Lung ultrasound in respiratory distress syndrome: a useful tool for early diagnosis. Neonatology. 2008;94(1):52-59.

7- Liu J, Wang Y, Fu W, Yang CS, Huang JJ. Diagnosis of neonatal transient tachypnea and its differentiation from respiratory distress syndrome using lung ultrasound. Medicine 2014; 93(27): e197.

8- Shui-Wen Chen, Wei Fu, Jing Liu, and Yan Wang. Routine application of lung ultrasonography in the neonatal intensive care unit. Medicine 2017 ; 96:102. 